\title{
REVISIÓN - EFECTO DEL SISTEMA DE VULCANIZACIÓN EN LA RED ENTRECRUZADA Y EN LA REACCIÓN QUÍMICA DE VULCANIZACIÓN DEL CAUCHO NATURAL
}

\author{
$\triangle$ William URREgo-YePES ${ }^{1}$ \\ SANDRA M. VELÁSQUEZ-RESTREPO ${ }^{2}$ \\ DiEGo H. GIRALDO-VÁSQUEZ ${ }^{3}$ \\ JuAN CARLOS POSADA CORREA ${ }^{4}$
}

\section{RESUMEN}

Se presenta una revisión sobre el efecto del sistema de vulcanización en la estructura formada durante la reacción de vulcanización, en la cinética de la reacción y en las propiedades de formulaciones que emplean caucho natural. Se encontró que aún existen aspectos por investigar dada la variedad de acelerantes, proporciones acelerante/azufre, tipos de caucho natural y formulaciones que emplean caucho natural. La mayor parte de la literatura evalúa la reacción de vulcanización mediante reometría de vulcanización y calorimetría diferencial de barrido (DSC), pero estas técnicas no permiten identificar el tipo de enlaces sulfídicos formados ni la densidad de enlaces entrecruzados. La temática es de gran interés científico e industrial pues el caucho natural es el caucho más comercializado a nivel mundial, y su vulcanización se realiza en la gran mayoría de las formulaciones empleando azufre como agente entrecruzante.

PALABRAS CLAVES: Caucho natural, sistema de vulcanización, reacción de vulcanización, red entrecruzada, agente vulcanizante, acelerantes

1 Ingeniero de Materiales, Magíster en ingeniería, Docente del programa de ingeniería de producción, Instituto Tecnológico Metropolitano (ITM). Medellín, Colombia.

2 Bioingeniera, Magíster en ingeniería, Líder de Innovación y desarrollo tecnológico-SENNOVA del Centro de Diseño y Manufactura del Cuero, Servicio Nacional de Aprendizaje (SENA). Itagüí, Colombia.

3 Ingeniero Mecánico, Magíster en ingeniería, Docente del programa de ingeniería de materiales, Universidad de Antioquia. Medellín, Colombia.

4 Ingeniero Industrial, Magíster en ingeniería, Docente del programa de ingeniería de producción, Instituto Tecnológico Metropolitano (ITM). Medellín, Colombia.

Autor de correspondencia: Urrego-Yepes, W. (William): Instituto Tecnológico Metropolitano (ITM). Calle 73 No. 76a-354, Medellín, Colombia. C.P.: 050034 Tel.: +574405100 Ext. 5290. Correo electrónico: williamurrego@ itm.edu.co
Historia del artículo:

Artículo recibido: 24-VII-2017 / Aprobado: 26-IV-2018

Disponible online: 30 de marzo de 2018

Discusión abierta hasta abril de 2019 


\section{REVIEW- EFFECT OF THE VULCANIZATION SYSTEM IN THE CROSSLINKED AND IN THE CHEMICAL VULCANIZATION REACTION OF NATURAL RUBBER}

\section{ABSTRACT}

This work presents a review on the effect of the vulcanization system on the crosslinked structure formed during vulcanization reaction and the properties of natural rubber-based materials. These topics have been subject of research because of the wide variety of accelerants, accelerant/sulphur ratios, types of natural rubber and natural rubber-based formulations. Most of the studies use rheometry and differential scanning calorimetry (DSC) for evaluating natural rubber vulcanization, but both techniques do not provide information about the type of sulfidic crosslinks or the crosslinks density. The theme is of great scientific and industrial interest because natural rubber is the most traded rubber worldwide, and its vulcanization is carried out in the vast majority of formulations using sulfur as crosslinking agent.

KEYWORDS: Natural rubber, vulcanization system, vulcanization reaction, crosslinked network, vulcanizing agent, accelerators.

\section{REVISÃO - EFEITO DO SISTEMA DE VULCANIZAÇÃO NA REDE DE LIGAÇÕES CRUZADAS E A REAÇÃO QUÍMICA DE VULCANIZAÇÃO DE BORRACHA NATURAL}

\section{RESUMO}

Este trabalho apresenta uma revisão sobre o efeito do sistema de vulcanização na estrutura formada durante a reação de vulcanização, na cinética da reação e nas propriedades das formulações que utilizam borracha natural. Esses tópicos tem sido objeto de pesquisa devido à grande variedade de acelerantes, proporções acelerante/enxofre, tipos de borracha natural e formulações baseadas em borracha natural. A maioria dos estudos utiliza reometria e calorimetria de varredura diferencial (DSC) para avaliar a vulcanização da borracha natural, mas ambas as técnicas não fornecem informação sobre o tipo de ligações sulfídicas ou a densidade de reticulação. 0 tema é de grande interesse científico e industrial porque a borracha natural é a borracha mais comercializada em todo o mundo, e sua vulcanização é realizada na grande maioria das formulações usando o enxofre como agente de reticulação.

PALAVRAS-CHAVE: Borracha natural, sistema de vulcanização, reação de vulcanização, rede de ligações cruzadas, agente de vulcanização, aceleradores.

\section{INTRODUCCIÓN}

Los materiales poliméricos se clasifican en termoplásticos, termoestables y elastómeros, según su estructura, comportamiento térmico y mecánico (Akay M., 2012). Los elastómeros, conocidos en español como cauchos (Fernández Torres, 2012) o hules (Martinez Valdés, 2012) son materiales que tienen como principal característica su capacidad de recuperar sus dimensiones originales después de haber sido sometidos a grandes deformaciones, presentando de esta manera un apreciable comportamiento elástico que los diferencia de los demás 
materiales de interés industrial (Akiba and Hashim, 1997; De Snaijer and Yuen, 2016).

Aproximadamente el $42 \%$ del caucho consumido en el mundo corresponde al caucho natural, obtenido mediante la extracción del látex presente en la corteza del árbol Hevea brasiliensis, mientras que los cauchos sintéticos son derivados del petróleo (Krishnan, 2015). El caucho natural posee una gran variabilidad en sus propiedades, esto se debe a factores como el tipo de clon del árbol del cual es extraído, las condiciones climáticas de la zona de cultivo, la configuración cis o trans que presente el poliisopreno que constituye entre el 94 y el $96 \%$ en peso del caucho natural, el contenido de contaminantes, sustancias orgánicas presentes en los vasos lactíferos de los árboles Hevea brasiliensis, como los principales factores (Akiba and Hashim, 1997; Ohm, 1990; Priyadarshan, 2017; Wren, 1960). Para conocer la configuración química del isopreno, el contenido de cenizas y demás componentes presentes en el caucho natural antes de ser mezclado con los ingredientes de la formulación del compuesto final, se emplean algunas técnicas de caracterización espectroscópicas y calorimétricas. Estos análisis sirven como control de calidad de la materia prima usada para la fabricación de productos que serán empleados en industrias como automovilística, aeronáutica y naval (B.I Gengrinovich, 1954; Davies, 1986; Fernández et al., 2006; Kuptsov and Zhizhin, 1998; Nallasamy and Mohan, 2004; Roland, 2004; Urrego et al., 2012).

Los productos de caucho natural pueden fabricarse a partir del material en estado sólido, luego de coagular el látex y procesar material coagulado, o empleando la suspensión coloidal de látex estabilizado; en cualquiera de los dos casos, se emplean diferentes aditivos que deben ser incorporados en la matriz de caucho natural. Cuando se fabrican productos a partir de látex, los ingredientes deben ser incorporados en forma de suspensión, mientras que en la fabricación de productos a partir del caucho natural sólido, se adicionan los diferentes ingredientes en estado sólido o en el estado líquido en el caso de los ayudantes de proceso (Mark et al., 2013;
Ohio, 1994). A continuación, se presentan los ingredientes empleados en la formulación de productos de caucho y los métodos empleados para su incorporación a la matriz de caucho.

\section{FORMULACIÓN DE COMPUESTOS}

\section{DE CAUCHO NATURAL, MEZCLADO E} INCORPORACIÓN DE LOS ADITIVOS EN

\section{LA MATRIZ DE CAUCHO NATURAL}

En la industria del caucho la receta o formulación, es decir, la elección de los componentes de la mezcla y las proporciones en que se usarán, se define con base en las características del compuesto de caucho, pero debe tenerse presente que los procesos de mezclado y de curado del compuesto también influyen en las propiedades del material elaborado (Dick, 2011).

Las formulaciones de compuestos de caucho pueden incluir entre 5 y 7 ingredientes para formulaciones básicas definidas por estándares como los de la American Society for Testing of Materials (ASTM, 2012; ASTM 2013), pero formulaciones comerciales pueden emplear hasta 23 ingredientes, entre los cuales se destacan la matriz de caucho, activadores, acelerantes, agentes de vulcanización como azufre y peróxidos, cargas reforzantes, ayudantes de proceso como agentes peptizantes o plastificantes, antidegradantes, pigmentos, entre otros (Dick, 2011; Lloyd, 1991; Ohio, 1994).

En las formulaciones de caucho la proporción de cada ingrediente se calcula tomando como referencia 100 partes de caucho (phr, siglas del término en inglés parts per hundred rubber). Es así como si la formulación considera dos, tres o más tipos de cauchos, la suma de todas las partes de caucho debe ser establecida como $100 \mathrm{phr}$. El resto de ingredientes se calcula con referencia a esas 100 partes de todos los cauchos de la formulación (Dick, 2011). En la Tabla 1 se presenta una formulación empleada para una banda de rodadura radial. 
TABLA 1. FORMULACIÓN DE CAUCHO PARA UNA BANDA DE RODADURA RADIAL DE UNA LLANTA (DICK, 2011)

\begin{tabular}{l|c}
\multicolumn{1}{c|}{ Ingrediente } & $\begin{array}{c}\text { Partes por cien de } \\
\text { caucho (PHR) }\end{array}$ \\
\hline SBR 1502 (Caucho sintético) & 80 \\
\hline SIR 20 (Caucho natural) & 20 \\
\hline N299 negro de humo & 65 \\
\hline Aceite nafténico & 10 \\
\hline TMQ (antioxidante) & 1 \\
\hline 6PPD (Diamina antiozonante) & 3 \\
\hline Mezcla de Cera & 2 \\
\hline Óxido de zinc & 5 \\
\hline Ácido esteárico & 1 \\
\hline TBBS (Sulfenamida acelerador) & 1.2 \\
\hline Azufre (Agente vulcanizante) & 2.5 \\
\hline
\end{tabular}

El azufre, por su reactividad y bajo costo, es el agente vulcanizante empleado preferentemente para mezclas basadas en caucho natural (Coran, 2013).

Los sistemas de vulcanización con azufre se clasifican en tres grupos: vulcanización convencional (CV), vulcanización semi eficiente (SEV) y vulcanización eficiente (EV), según la relación entre las proporciones de acelerante y azufre en la formulación. Por ejemplo en el sistema de vulcanización eficiente se emplean bajas cantidades de azufre, o incluso cero cuando se emplean acelerantes donadores de azufre, y altas cantidades de acelerantes, mientras que en un sistema de vulcanización convencional se emplean altas proporciones de azufre $y$ bajas cantidades de acelerantes (Akiba and Hashim, 1997; Datta, 2002; Dick, 2011).

En la Tabla 2 se muestran las proporciones de acelerante y azufre empleadas en los diferentes sistemas de vulcanización.

Para el proceso de pesaje de los ingredientes de la formulación se deben establecer tolerancias de acuerdo con estándares como la norma ASTM D 3182 (ASTM D3182, 2013), siendo críticas las medidas de los pesos de los ingredientes que están pre- sentes en pequeñas proporciones como el azufre y los acelerantes (Dick, 2011), lo cual puede conllevar a variaciones significativas entre lotes de producción o incluso en diferentes zonas de una misma mezcla, lo cual puede conllevar a variaciones en la red entrecruzada en un mismo producto.

TABLA 2. PROPORCIONES DE AZUFRE Y ACELERANTES EN SISTEMAS DE VULCANIZACIÓN CONVENCIONAL, SEMI EFICIENTE Y EFICIENTE

\begin{tabular}{c|c|c|c}
$\begin{array}{c}\text { Tipo de } \\
\text { sistema }\end{array}$ & $\begin{array}{c}\text { Azufre S } \\
\text { (phr) }\end{array}$ & $\begin{array}{c}\text { Acelerante } \\
\text { A (phr) }\end{array}$ & $\begin{array}{c}\text { Relación } \\
\text { A/S }\end{array}$ \\
\hline Convencional & $2.0-3.5$ & $1.2-0.4$ & $0.1-0.6$ \\
\hline Semieficiente & $1.0-1.7$ & $2.5-1.2$ & $0.7-2.5$ \\
\hline Eficiente & $0.4-0.8$ & $5.0-2.0$ & $2.5-12$ \\
\hline
\end{tabular}

Una vez pesados, los diferentes ingredientes de la formulación se incorporan en la matriz de caucho en equipos de procesamiento conocidos como mezcladores de caucho. Comúnmente se emplean tres tipos de mezcladores de caucho: molinos abiertos de dos rodillos, mezcladores internos tipo Banbury, y mezcladores internos miniatura. Las velocidades de mezclado y la dispersión de los ingredientes en el compuesto de caucho pueden ser diferentes de acuerdo al equipo de mezclado empleado (Dick, 2011; WHITE, 1994).

Es importante establecer el orden de adición de los diferentes ingredientes en la matriz de caucho, pues las propiedades del compuesto dependen de ese orden, llamado esquema de mezclado. Se ha demostrado que las propiedades reológicas y el comportamiento físico-mecánico del compuesto de caucho natural pueden verse influenciadas por factores como el tipo de mezclador empleado y el esquema de mezclado (Akinlabi et al., 2006; Pinchuk et al., 2001).

Luego de tener el compuesto de caucho mezclado con todos ingredientes establecidos en la formulación, se deben evaluar las condiciones de vulcanización del material, proceso que permite definir condiciones de procesamiento del material. 


\section{VULCANIZACIÓN DE CAUCHO}

\section{NATURAL}

\section{Reacción de vulcanización}

Los elastómeros crudos o sin vulcanizar presentan pobres propiedades relacionadas con su resistencia físico-mecánica, y presentan un comportamiento preferentemente plástico al aplicarle esfuerzos mecánicos, características de poco interés para la industria (Coran, 2013). El proceso de vulcanización, también llamado curado en algunas referencias de la literatura puede definirse como el mecanismo químico mediante el cual se genera un entrecruzamiento entre las cadenas moleculares del caucho, requiriendo agentes de entrecruzamiento como azufre o compuestos sulfurados, y en otros casos elementos como peróxidos. Los mecanismos presentes en la reacción de vulcanización y la red entrecruzada formada, y por ende las propiedades tecnológicas del material, dependen de forma significativa de si se elige azufre o se eligen peróxidos como agente de vulcanización
(Akiba \& Hashim, 1997) (Coran, 2013)(Coran, 1994; Saville and Watson, 1963).

En la Figura 1 se esquematiza la manera como se altera la estructura del caucho como resultado del proceso de vulcanización con azufre, (Coran, 2013)(Coran, 1994).

Figura 1. Red molecular del caucho antes y después del proceso de vulcanización con azufre (Coran, 2013). Traducción libre de los autores a partir de la figura original

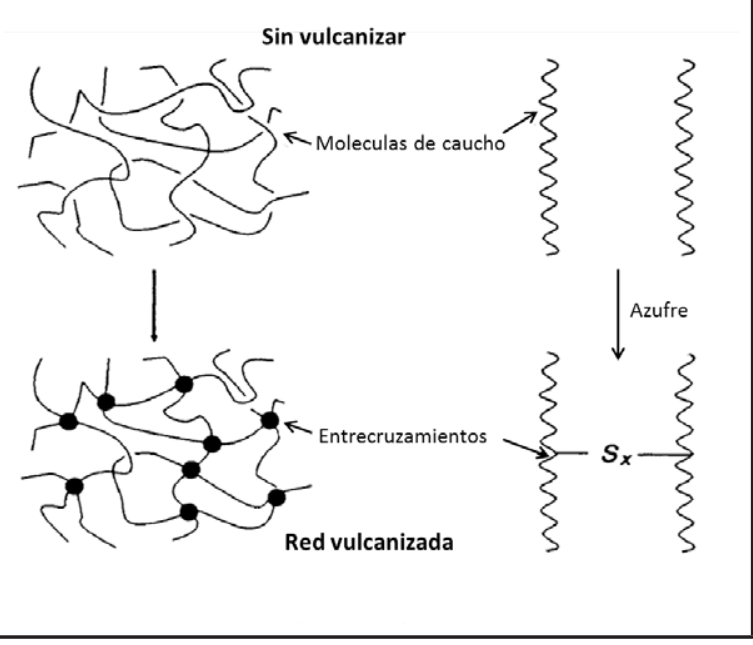

Figura 2. Posibles configuraciones en la estructura del caucho natural vulcanizado con azufre. (Akiba and Hashim, 1997). Traducción libre de los autores a partir de la figura original

$\underline{\text { Red entrecruzada }}$

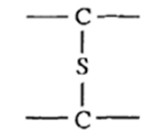

Monosulfidico

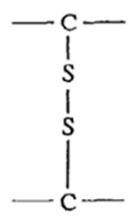

Disulfidico

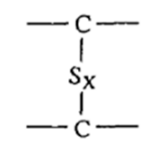

Polisulfidico $x>2$

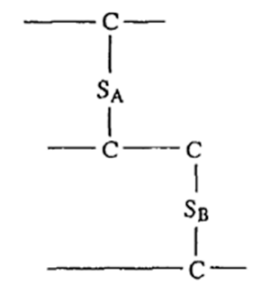

Vecinos multifuncionales

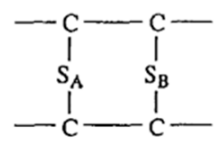

Vecinos
Otras modificaciones

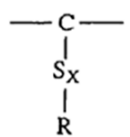

Grupo pendiente ( $R=$ Residuo del acelerante)

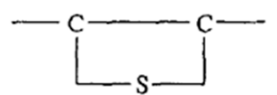

Azufres ciclicos

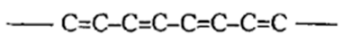

Insaturaciones conjugadas<smiles>CC=CCCCCC=CC</smiles>

Isomerización cis/trans 
Durante la reacción de vulcanización se genera la unión química entre la estructura molecular del caucho con los átomos de azufre, mediante esta unión se pueden generar puentes elásticamente activos y al mismo tiempo otros tipos de enlaces entre el caucho y el azufre que no lo son; los diferentes tipos de enlaces que se podrían generar entre el caucho natural y los átomos de azufre durante el proceso de vulcanización son mostrados en la Figura 2.

La temperatura que alcanza el caucho durante el procesamiento incide de manera directa en velocidad de vulcanización, pues se trata de una reacción activada térmicamente. Una vez se establece una temperatura o un rango de temperaturas para realizar el proceso de vulcanización, deben elegirse los tiempos de vulcanización para que el material alcance la densidad de enlaces entrecruzados de interés para el producto. Para una concentración e ingredientes del sistema de vulcanización dados, la red puede alcanzar una densidad máxima de enlaces entrecruzados, de tal manera que una vez se desarrolla el proceso de vulcanización la proporción entre la densidad de enlaces lograda realmente con respecto a la máxima teórica se denomina grado de vulcanización. El grado de vulcanización será entonces cero para un compuesto totalmente crudo en el cual no ha iniciado la reacción de entrecruzamiento, y será uno cuando el compuesto alcanzó la máxima densidad de entrecruzamiento posible con el sistema de vulcanización empleado en la formulación. Sólo cuando se emplean las condiciones de temperatura y tiempo de vulcanización adecuados, se alcanzará en el producto un grado de vulcanización de uno. La temperatura y el tiempo de proceso determinan el grado de vulcanización alcanzado, el cual a su vez determina las prestaciones físicas y químicas del producto en servicio.

Para medir el avance del grado de vulcanización se pueden emplear varias técnicas instrumentales entre las cuales se destacan la reometría de vulcanización y la calorimetría diferencial de barrido (DSC por sus siglas en inglés). También puede emplearse la evaluación de propiedades mecánicas como dureza y módulo de elasticidad cuando el material es vulcanizado a diferentes tiempos a una misma temperatura, pero es una metodología mucho más engorrosa e imprecisa que la reometría de vulcanización o la DSC.

La determinación de la cinética de vulcanización mediante DSC se basa en la naturaleza exotérmica de la reacción de vulcanización; los datos de entalpía proporcionados por el equipo permiten calcular el calor liberado por la muestra desde el inicio de la reacción, de tal manera que se monitorea el avance del grado de vulcanización desde que inicia la liberación de calor hasta que finaliza.

La medición mediante DSC no permite discriminar si los enlaces formados son elásticamente activos o no (Marzocca et al., 2004; Peres et al., 2006). El DSC permite además realizar seguimiento de la reacción de vulcanización de forma isotérmica o no isotérmica, una versatilidad que es muy útil para establecer la cinética de vulcanización bajo diferentes ciclos térmicos, lo cual se aproxima mejor a la manera como se da la reacción en los procesos industriales.

La técnica conocida como reometría de vulcanización monitorea el cambio en el torque que se aplica a la muestra para generarle una deformación constante, en condiciones isotérmicas, siendo la de disco móvil sin rotor bajo norma ASTM D5289 la más empleada en la actualidad (ASTM D5289, 2012, p. 11). Al avanzar la reacción de vulcanización se presenta un aumento en el torque debido a que la rigidez del material se incrementa de manera proporcional a la densidad de enlaces entrecruzados elásticamente activos entre las cadenas de caucho natural, Los puentes monosulfídicos, disulfídicos y polisulfídicos forman enlaces elásticamente activos, es decir, que aportan a la rigidez del caucho y por ende se requiere un mayor torque a medida que se forman más enlaces elásticamente activos. El tipo y proporción de cada tipo de enlace depende del sistema de vulcanización empleado, de la temperatura empleada durante la reacción de vulcanización, y por ende determinan las propiedades del compuesto de caucho natural vulcanizado con azufre. 
Una ventaja de monitorear la reacción de vulcanización mediante reometría de vulcanización es que permite medir el torque incluso después de finalizar la vulcanización, de tal manera que se evidencien fenómenos como reversión, que consiste en la disminución en la rigidez del compuesto vulcanizado como resultado de la desulfurización en los enlaces formados en el caucho natural (Akiba and Hashim, 1997; Coran, 2013).

Tanto la reometría de vulcanización como la DSC permiten establecer los tiempos de inducción de la reacción de vulcanización, es decir, el tiempo necesario para que se formen los primeros enlaces elásticamente activos entre el caucho y el azufre en el caso de la reometría de vulcanización, y el momento en que se comienza a liberar calor como efecto de la vulcanización en el caso de la DSC. Estos tiempos dependen de los ingredientes empleados en la formulación, como por ejemplo los acelerantes y la estabilidad de los mismos, lo cual influye directamente en la energía de activación del proceso químico de vulcanización (Franck et al., 1962; Heideman, 2004; Morrison and Porter, 1984a).

Es pertinente tener en cuenta que la reacción de vulcanización con azufre puede presentarse incluso sin que se usen activadores y/o acelerantes. Estos ingredientes tienen como propósito principal modificar los tiempos necesarios para lograr un grado de vulcanización total. Por lo tanto, los mecanismos químicos presentes durante la reacción de vulcanización del caucho natural van a depender de la presencia, o no, de activadores y de acelerantes. Es así como se ha establecido que la reacción de vulcanización puede ser iónica o radicalaria, o una combinación de ambas, y se han propuesto mecanismos para la reacción de vulcanización tanto con azufre y acelerante sin activadores como con azufre, acelerante con activadores, diferenciando estos dos procesos principalmente por la formación de un complejo intermedio de zinc que influye directamente en los tiempos requeridos para que se dé reacción de vulcanización (Hauser and Sze, 1942; Morrison, 1984a, 1984b; Shelton and McDonel, 1960; Vergnaud and Rosca, 2009).
La adición de cargas reforzantes a las formulaciones de caucho natural también puede afectar la velocidad de la reacción de vulcanización, además de generar otro tipo de enlaces e interacciones adicionales con el caucho y con el resto de ingredientes empleados en la formulación, entre ellos los sistemas de vulcanización (Villars, 1957). Teniendo en cuenta que el negro de humo es la carga reforzante más empleada para en la industria del caucho, es relevante mencionar que esta carga puede interactuar con el caucho natural mediante uniones físicas y químicas. Algunos autores como Blokh et al, consideran la formación de enlaces sulfidicos intermedios entre los acelerantes y las partículas de negro de humo, al mismo tiempo que se generan interacciones con los carbonos presentes en la estructura molecular el caucho natural (Blokh and Yaroshevich, 1957).

Las etapas de la reacción de vulcanización con azufre pueden resumirse de forma general como se muestra en la Figura 3. Este esquema abarca de forma general los mecanismos de reacción iónica y radicalaria, permitiendo entender la vulcanización del caucho natural en presencia de azufre y acelerantes (Heideman, 2004; Morrison and Porter, 1984b).

La cinética del proceso de vulcanización puede ser establecida mediante el ajuste de modelos matemáticos a los resultados experimentales obtenidos por DSC o por reometría de vulcanización. Kamal y Sourour, por ejemplo, consideraron el ajuste a un modelo matemático de una reacción de curado de polímeros termoestables, estableciendo dos parámetros asociados a la velocidad de la reacción de curado y el orden de la reacción (Kamal and Sourour, 1973). Varios autores han empleado dicho ajuste matemático con datos experimentales obtenidos mediante DSC durante el proceso de curado de cauchos y termoestables (Jarny, 2000; Rabearison et al., 2010; Zapata et al., 2012). El mismo ajuste matemático puede ser utilizado con los datos experimentales obtenidos mediante reometría de vulcanización, específicamente para el proceso de vulcanización de cauchos (Mansilla et al., 2007; Marzocca, 2003; Marzocca et al., 2010). 
Figura 3. Vulcanización de caucho en presencia de azufre, acelerantes y activadores. La cadena de caucho es $\mathrm{R}$ y el residuo del acelerante es X (Heideman, 2004; Heideman et al., 2004). Traducción libre de los autores a partir de la figura original

Acelerantes y activadores

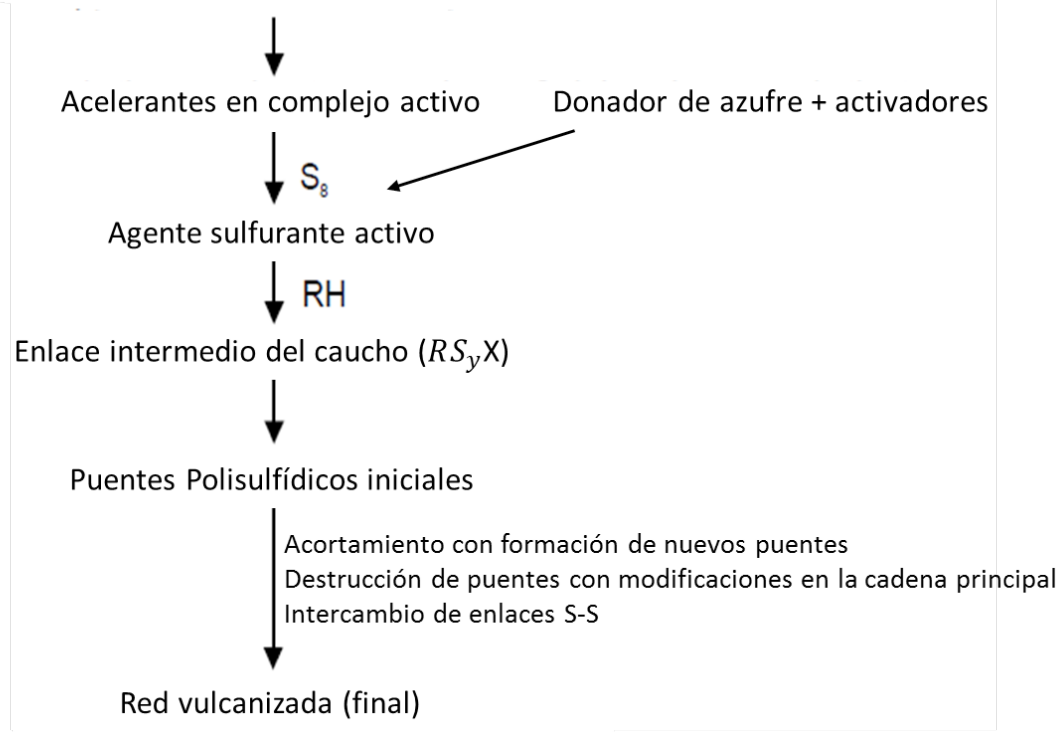

\section{Densidad de enlaces entrecruzados} en mezclas que emplean caucho natural

Los enlaces entrecruzados se forman a ciertos espaciamientos promedio a lo largo de las cadenas del caucho natural. La estructura alcanza densidades de entrecruzamiento que dependen de la cantidad de azufre y acelerante empleado en la formulación (Coran, 2013).

A medida que a reacción de vulcanización avanza, aumenta la densidad de entrecruzamiento; en la Figura 4 se indica el avance en el tiempo de la reacción de vulcanización mediante una reometría de vulcanización, pero debe advertirse que la técnica no permite cuantificar la densidad de entrecruzamiento sino que permite calcular el grado de vulcanización como la relación entre el torque en un momento dado y el valor máximo del torque, dado que cuando el torque alcanza este máximo es porque se alcanzó la máxima densidad de enlaces elásticamente activos (Coran, 2013).

Si se quiere medir la densidad de entrecruzamiento de manera cuantitativa, se pueden emplear varias técnicas analíticas experimentales, entre las que destacan el hinchamiento en solventes (Mansilla, 2012; Marzocca, 2007; Marzocca et al., 2010) y el ajuste del modelo de reptación a los resultados experimentales del comportamiento de un ensayo en tensión a bajas velocidades de deformación (Cerveny and Marzocca, 1999; Heinrich et al., 1988; Marzocca et al., 1997).

Para evaluar la densidad de entrecruzamiento mediante hinchamiento en solventes, se debe extraer inicialmente la fracción de caucho que no logró vulcanizar, esta fracción es conocida como la fracción sol; de esta manera se espera que la fase que quede en el producto sea la fase que logró vulcanizar, conocida como la fase gel, que corresponde a la fase que mantiene las características propias del material elástico dado el entrecruzamiento generado entre las cadenas (Allen and Bristow, 1963; Flory, 1942).

Se establece entonces, que la fracción "sol" del compuesto de caucho presenta solubilidad en un solvente seleccionado, cuando la energía libre de la mezcla es igual a cero de acuerdo a la Ecuación 1 (Ferry et al., 1945; Gee, 1943, 1942). 
Figura 4. Descripción de las curvas de vulcanización obtenida que pueden obtenerse por reometría. Puede observarse que existe una relación directa entre el torque que mide el equipo y la densidad de entrecruzamiento (Coran, 2013). Traducción libre de los autores a partir de la figura original

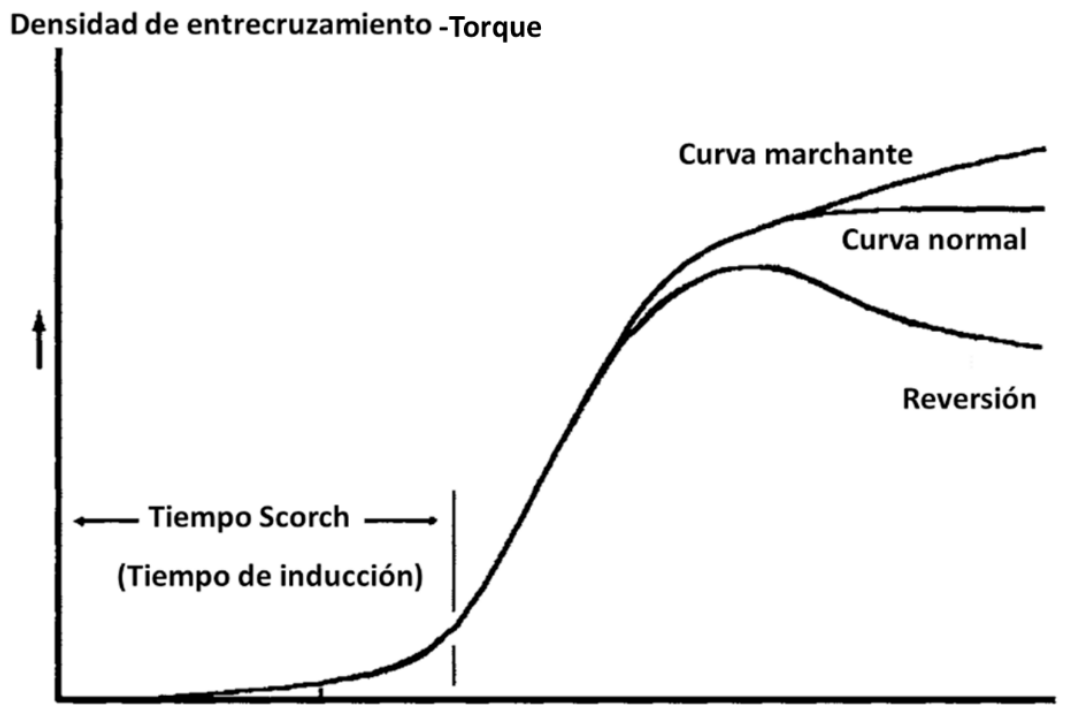

Tiempo de vulcanización

$$
\Delta G_{m}=\Delta H_{m}-T \cdot \Delta S_{m}
$$

Donde, $\Delta G_{m}$ es la energía libre de la mezcla, $\Delta H_{m}$ es la entalpía de mezcla, T es la temperatura y $\Delta S_{m}$ es la entropía de mezcla.

Para que la energía libre de la mezcla sea menor a cero, la entalpia de la mezcla debería ser igual a cero, y entonces haciendo uso de la ecuación de Hildebrand (Ecuación 2) podemos establecer que la solubilidad entre el caucho sin vulcanizar y un solvente en particular se presentará cuando los parámetros de solubilidad del polímero y del solvente son similares (Ferry et al., 1945).

$$
\Delta H_{M}=\phi_{1} \phi_{2}\left(\delta_{1}-\delta_{2}\right)^{2}
$$

Donde $\phi_{1}, \phi_{2}, \delta_{1}$ y $\delta_{2}$ son las concentraciones y parámetros de solubilidad del polímero y del solvente respectivamente.

Luego de la extracción de la fase soluble del caucho -fracción sol-, el material que queda correspondiente a la fracción gel -fracción reticula- da-, es llevado a un proceso de hinchamiento en un solvente, donde se genera un aumento en las dimensiones del material como consecuencia de la deformación elástica generada en la estructura químicamente entrecruzada. Flory y Rehner establecieron una relación que representa el estado de hinchamiento en equilibrio con el peso molecular de las cadenas del polímero entre los puntos de enlace de los puentes entrecruzados $\left(\mathrm{M}_{\mathrm{c}}\right)$, y luego a partir de este peso molecular se puede conocer la densidad de entrecruzamiento del material (Flory and Rehner, 1943a, 1943b).

Como se mencionó previamente, la densidad de entrecruzamiento es marcadamente influyente en las propiedades físico-mecánicas del caucho vulcanizado. En la Figura 5 se muestra el efecto que tiene la densidad de entrecruzamiento en propiedades del caucho vulcanizado como la dureza, el esfuerzo a la tensión, resistencia al corte, resistencia a la fatiga, rigidez, recuperación elástica, histéresis y deformación permanente. 
Figura 5. Efecto de la densidad de entrecruzamiento en algunas propiedades tecnológicas del caucho vulcanizado (Coran, 2013). Traducción libre de los autores a partir de la figura original

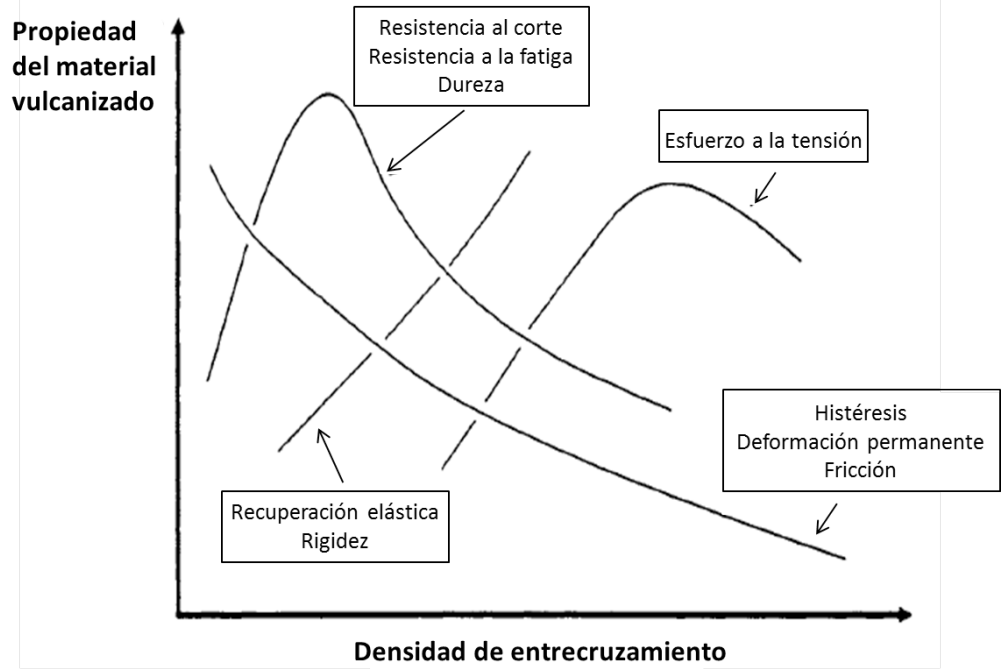

\section{INFLUENCIA DEL SISTEMA} DE VULCANIZACIÓN EN LA RED

\section{ENTRECRUZADA Y EN LA REACCIÓN QUÍMIICA DE VULCANIZACIÓN}

Las estructuras de los cauchos vulcanizados con azufre han sido determinadas mediante técnicas espectroscópicas como infrarrojo (IR), ultravioleta (UV), Raman y mediante algunos métodos analíticos y fisicoquímicos. Adicionalmente, los estudios realizados mediante resonancia magnética nuclear (NMR) también han ayudado a establecer los posibles tipos de enlaces que se pueden generar en la red entrecruzada de cauchos dienos de alto grado de insaturación, como es el caso del caucho natural (Akiba and Hashim, 1997).

Se ha encontrado entonces que la cantidad de cada tipo de enlace sulfídico presente en los cauchos vulcanizados con azufre depende de factores como: el tipo de caucho empleado, la formulación del compuesto del caucho, pero más especialmente el sistema de vulcanización, agentes auxiliares y algunas condiciones del proceso de vulcanización como la temperatura empleada y el tiempo de curado. Se ha identificado que los puentes monosulfídicos se forman en una formulación de manera preferente cuando se emplean altas cantidades de acelerante comparado con la cantidad de azufre o de donadores de azufre (Akiba and Hashim, 1997; Mukhopadhyay et al., 1978; Mukhopadhyay and De, 1977).

En la Tabla 3 se presenta de forma resumida el tipo de enlace sulfídico formado en el caucho natural de acuerdo con el sistema de vulcanización (SV) empleado, así como la influencia del SV en algunas propiedades de los compuestos vulcanizados como la resistencia a la cristalización a bajas temperaturas, resistencia al envejecimiento térmico, resistencia a la reversión y comportamiento frente a deformaciones permanentes, propiedad tecnológica denominada como compression set.

De acuerdo con la Tabla 3, se puede establecer entonces que en el caso de formulaciones de caucho natural con azufre se elige el tipo de sistema de vulcanización según el tipo de enlaces sulfídicos entrecruzados que se deseen en el compuesto, teniendo como referencia la estabilidad térmica que se requiere para la aplicación, y por ende el comportamiento frente a la reversión, al envejecimiento y la respuesta en el ensayo de compression set. 
TABLA 3. TIPOS DEENLACESY PROPIEDADES DE CAUCHOS NATURALES SEGÚN ELTIPO DE SISTEMA DEVULCANIZACIÓN CON AZUFRE EMPLEADO (AKIBA AND HASHIM, 1997)

\begin{tabular}{l|c|c|c|} 
& Convencional & Semi eficiente & Eficiente \\
\hline Entrecruzamientos poli y disulfidicos (\%) & 95 & 50 & 20 \\
\hline Entrecruzamientos monosulfidicos & 5 & 50 & 80 \\
\hline Concentración de azufre cíclico & Alta & Media & Baja \\
\hline $\begin{array}{l}\text { Resistencia a la cristalización a bajas } \\
\text { temperaturas }\end{array}$ & Alta & Media & Baja \\
\hline Resistencia al envejecimiento por calor & Baja & Media & Alta \\
\hline Resistencia a la reversión & Baja & Media & Alta \\
\hline Compression set, $22 \mathrm{~h} \mathrm{a} 70^{\circ} \mathrm{C}(\%)$ & 30 & 20 & 10 \\
\hline
\end{tabular}

Independientemente del tipo de formulación empleada, la temperatura establecida durante el proceso y el tiempo de vulcanización pueden modificar la estructura entrecruzada, ya que los enlaces polisulfídicos poseen baja resistencia térmica, mientras que los enlaces monosulfídicos poseen una mayor estabilidad térmica (Mukhopadhyay et al., 1978). Si se emplea un sistema convencional en una formulación de caucho natural se espera que durante el proceso de vulcanización el compuesto presente reversión, debido a la degradación de los enlaces polisulfídicos y la formación de enlaces monosulfídicos cíclicos, es decir, se disminuye la red entrecruzada y por lo tanto disminuye la densidad de entrecruzamiento del compuesto (Akiba and Hashim, 1997; Loo, 1974).

En la Figura 6 se observa la dependencia de la densidad de entrecruzamiento frente a la temperatura de un compuesto de caucho formulado con un SV convencional.

Figura 6. Efecto de la temperatura en la densidad de entrecruzamiento de un compuesto de caucho elaborado con un SV convencional con azufre (Loo, 1974). Traducción libre de los autores a partir de la figura original

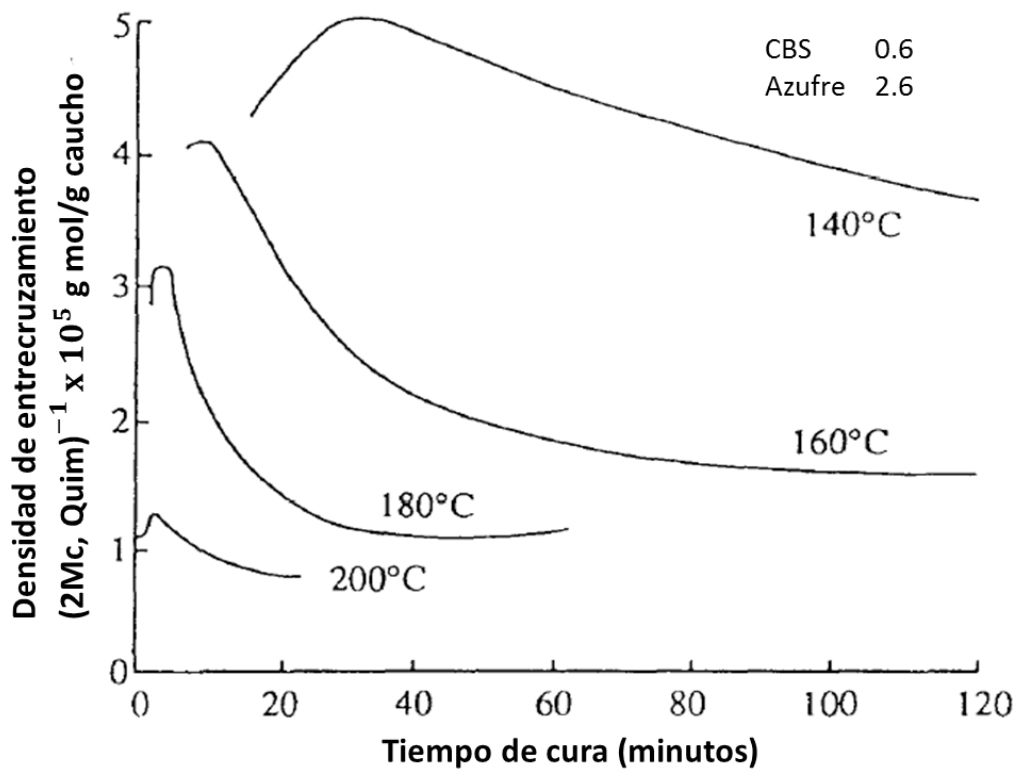


Cuando se emplean largos tiempos de vulcanización se genera una disminución de la densidad de entrecruzamiento, pero adicionalmente se evidencia que aumentar la temperatura en el proceso de vulcanización disminuye la densidad de entrecruzamiento máxima a la que puede llegar el compuesto, y la degradación de la estructura entrecruzada comienza más rápido.

En cuanto al comportamiento mecánico, es relevante mencionar que cuando se evalúa el efecto de los sistemas de vulcanización en la elasticidad y las propiedades mecánicas de los compuestos de caucho natural vulcanizados con azufre, se encuentra que los enlaces polisulfídicos presentan una mayor rigidez y resistencia mecánica comparados con los enlaces monosulfídicos.

La cinética de la reacción de vulcanización también se verá modificada debido a la presencia de una mayor o menor proporción de acelerante y azufre, encontrando que el tiempo de inducción aumenta a medida que aumenta el contenido de acelerante en la formulación. Este fenómeno se presenta debido a los mecanismos de descomposición del acelerante, los mecanismos de reacción del acelerante con el azufre para formar el precursor de reacción y finalmente la formación de la red entrecruzada en el caucho (Gradwell and Merwe, 1999a, 1999b; Heideman, 2004; Heideman et al., 2004).

En la Figura 7 se esquematizan los mecanismos de descomposición del acelerante N-tert-butil2-benzotiazol sulfonamida (TBBS) y las posibles reacciones con el azufre presente en la formulación; estas reacciones son las que finalmente permiten aumentar el tiempo de inducción de la reacción para una formulación con TBBS.

Figura 7. Mecanismos de reacción del TBBS y azufre durante la primera etapa de un proceso de vulcanización (Heideman et al., 2004). Traducción libre de los autores a partir de la figura original

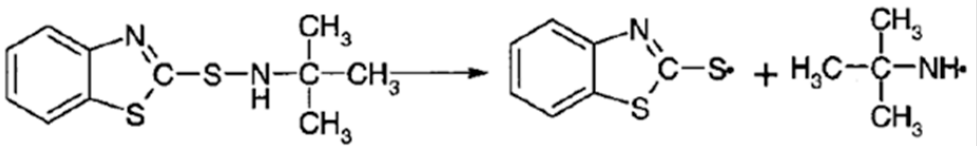

(TBBS)
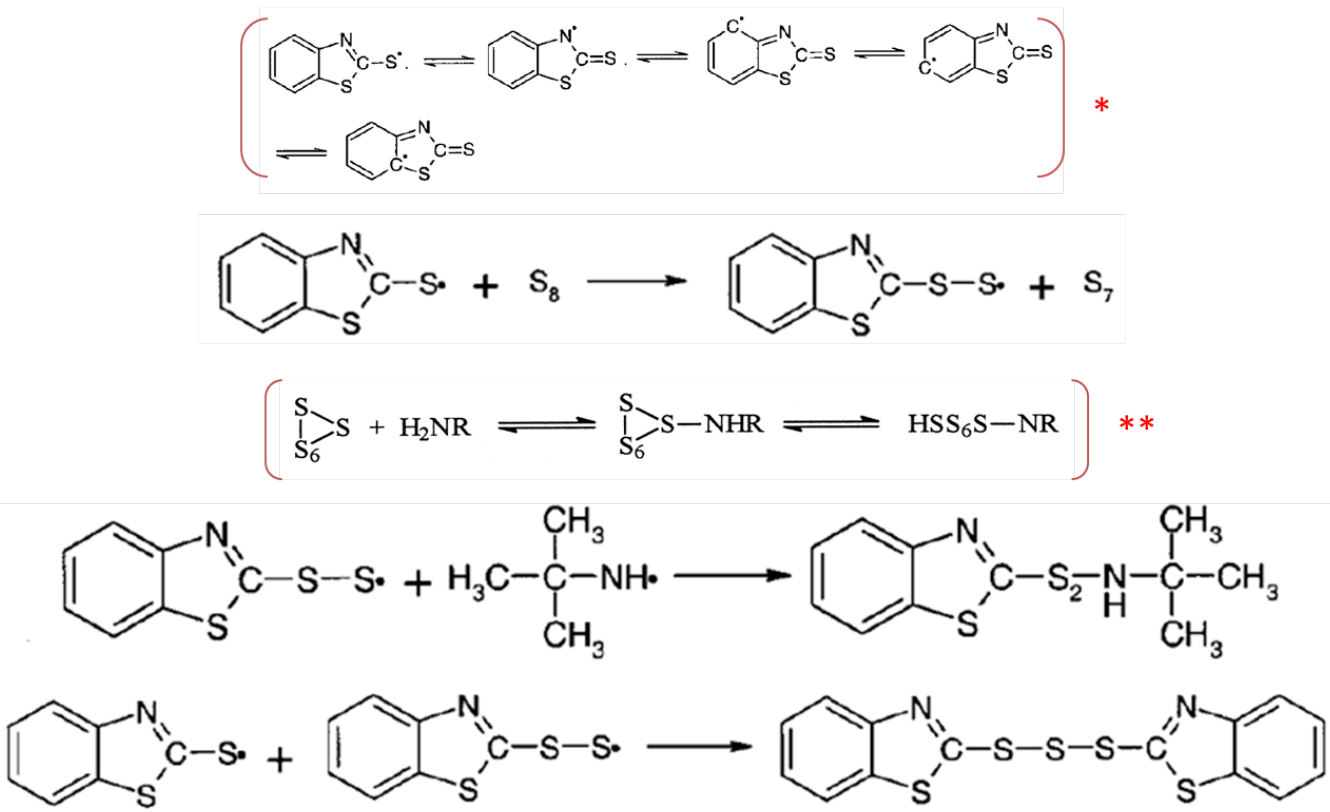
Figura 8. Mecanismos de formación de la red entrecruzada y del precursor de entrecruzamiento durante la reacción de vulcanización (Heideman et al., 2004). Traducción libre de los autores a partir de la figura original
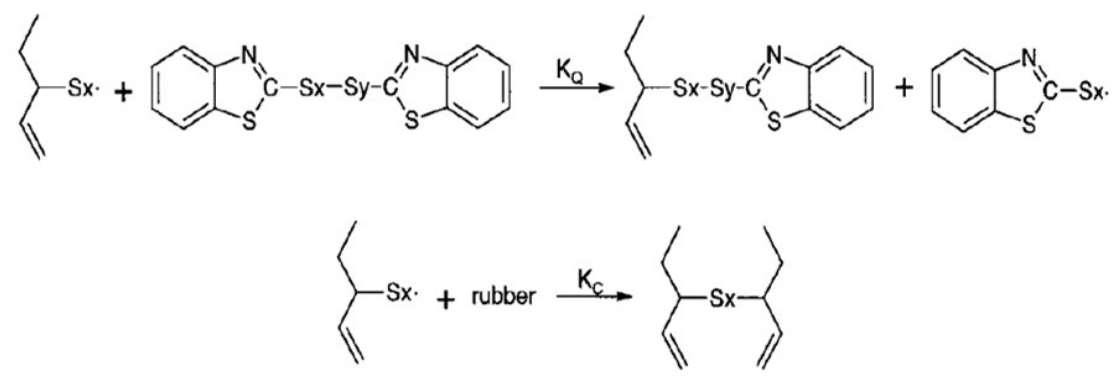

Figura 9. Efecto de los acelerantes en el tiempo de inducción o tiempos scorch y en la velocidad de vulcanización (Coran, 2013). Traducción libre de los autores a partir de la figura original

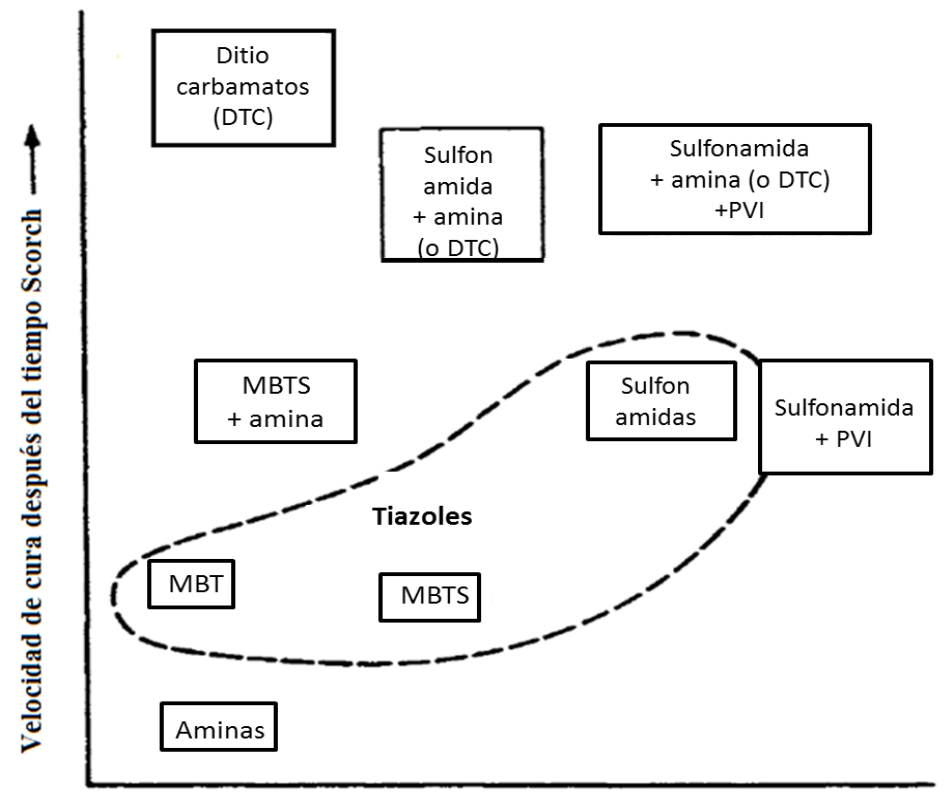

Tiempo de inducción o Scorch

De acuerdo con la Figura 7, el TBBS se descompone formando inicialmente el radical mercapto, luego este reacciona con el azufre y se une a una molécula radical de amina formando una nueva molécula de TBBS disulfídica o podría reaccionar con otro radical mercapto y de esta manera formar un molécula de 2-Mercaptobenzotiazol-disulfide (MBTS) trisulfídica (Gradwell and Merwe, 1999a; Mayer, 1977).

En la Figura 8 se puede observar la etapa siguiente del proceso de vulcanización con azufre y TBBS, donde las moléculas formadas en etapas an- teriores reaccionan con las cadenas de caucho para formar precursores de entrecruzamiento con una velocidad de formación establecida por la constante cinética de reacción $\mathrm{K}_{\mathrm{Q}}$. La formación del precursor de entrecruzamiento es mucho más rápida que la formación de la red de entrecruzada del caucho, ya que la constante cinética $\mathrm{K}_{0}$ es mucho mayor que $\mathrm{K}_{\mathrm{C}^{\prime}}$ donde $\mathrm{K}_{\mathrm{C}}$ es la constante cinética de la reacción de entrecruzamiento de la cadenas de caucho natural, por lo tanto la formación del precursor de entrecruzamiento aporta también al tiempo de inducción (Heideman et al., 2004). 
En la Figura 9 se muestra el efecto de diferentes acelerantes en el tiempo de inducción y en la velocidad de la reacción de vulcanización de compuestos de caucho. Entonces los tiempos y la reacción de vulcanización pueden ser modificados de acuerdo con el SV empleado, sin embargo, es importante definir los límites de cada uno de los acelerantes empleados en la formulación.

\section{CONCLUSIONES}

Es pertinente comprender el efecto del sistema de vulcanización con azufre en el tipo de estructura entrecruzada que se forma en los cauchos naturales durante la vulcanización, dada la complejidad de los mecanismos de la reacción y el efecto de diversas variables como temperatura del material, cantidad y tipo de cargas reforzantes y agentes activadores empleados en la formulación. El análisis de la literatura sobre el tema demuestra que aún existen aspectos por estudiar, dado que existe un amplio rango de formulaciones que emplean caucho natural, de tal manera que son numerosas las posibles combinaciones de acelerantes, contenidos de azufre, proporciones acelerantes/azufre y tipos de caucho natural puesto que es un material extraído de una planta que es susceptible a las condiciones de cultivo.

Se evidencia que la elección de los ingredientes del sistema de vulcanización con azufre, así como las proporciones en que se emplean en las formulaciones de caucho natural, determina la eficiencia de la reacción de vulcanización y el tipo de enlaces sulfídicos formados. Este aspecto es de alta relevancia dado que los enlaces mono y disulfídicos, formados con altas relaciones acelerante/azufre, otorgan a los compuestos de caucho natural mayor estabilidad térmica y menor rigidez que los enlaces polisulfídicos, que se forman cuando la relación acelerante/azufre son bajas. Se trata de un fenómeno de alto interés científico e industrial, dado que el caucho natural es el caucho más comercializado a nivel mundial y su vulcanización se realiza en la gran mayoría de las formulaciones empleando azufre como agente entrecruzante.
Los estudios reportados han empleado preferentemente la reometría de vulcanización como técnica instrumental para estudiar la reacción de vulcanización, seguida de la calorimetría diferencial de barrido (DSC), pero con la limitante de que no permiten identificar el tipo de enlaces sulfídicos formados ni la densidad de enlaces entrecruzados. Estas técnicas son de ejecución es relativamente simple, sin embargo, la interpretación de los resultados obtenidos exige un conocimiento de la reacción de vulcanización por lo cual la revisión realizada en este trabajo es necesaria.

\section{AGRADECIMIENTOS}

Los autores queremos agradecer al Instituto Tecnológico Metropolitano (ITM), al Servicio Nacional de Aprendizaje (SENA) y a la Universidad de Antioquia (UdeA) por sus aportes institucionales.

\section{REFERENCIAS}

Akay M., 2012. Introduction To Polymer Sciense And Technology. 1 St Ed. Mustafa Akay Ventus Publ. ApS.

Akiba, M., Hashim, A.S., 1997. Vulcanization and crosslinking in elastomers. Prog. Polym. Sci. 22, 475-521.

Akinlabi, A.K., Okieimen, F.E., Egharevba, F., Malomo, D., 2006. Investigation of the effect of mixing schemes on rheological and physico-mechanical properties of modified natural rubber blends. Mater. Des. 27, 783-788. doi:10.1016/j.matdes.2005.01.007

Allen, P.W., Bristow, G.M., 1963. The gel phase in Natural Rubber. J. Appl. Polym. Sci. 7, 603-615.

ASTM D3182, 2013. Standard Practice for Rubber - Materials, Equipment, and Procedures for Mixing Standard Compounds.

ASTM D5289, 2012. Test Method for Rubber Property-Vulcanization Using Rotorless Cure Meters. ASTM International.

B.I Gengrinovich, 1954. Calorific and thermal properties of natural rubber in the oriented and non-oriented states. Rubber Chem. Technol. 95, 571-574.

Blokh, G.A., Yaroshevich, A.G., 1957. The interaction between carbon black and sulfur during vulcanization. Proceeding Acadmey Sci. USSR Phys Chem Sect 116, 583-586. 
Cerveny, S., Marzocca, A.J., 1999. Analysis of variation of molecular parameters of natural rubber during vulcanization in conformational tube model. II. Influence of sulfur/accelerator ratio. J. Appl. Polym. Sci. 74, 2747-2755. http://onlinelibrary.wiley.com/doi/10.1002/(SICI)10974628 (19991209) 74 :11\%3C274 7 : : A I D APP22\%3E3.0.CO;2-N/full.

Coran, A., 2013. 7 Vulcanization. Sci. Technol. Rubber 337. Datta, R.., 2002. Rubber curing systems. iSmithers Rapra Publishing. http://books.google.com/ books?hl=en\&lr=\&id=XoZa5t3_ogAC\&oi=fnd\&pg=P A3\&dq=\%22Evans,+Engelmann+\%26+Buckham $\% 2$ 2+\%22Research+(Caswell)\%22+\%22\%26+N+Tech nology+Ltd.+and+BIP+Chemicals $\% 22+\% 22$ Coates+ and+A.F.+Johnson, + IRC+in+Polymer\%22+\%22G.+W oods, $\% 22+\% 22$ Kirby, +Du+Pont+(U.K.) $\% 22+\& o t s=$ mu32fkpbF0\&sig=Nr_6AjWksG70toVPaZOXFiogeYY.

Davies, B., 1986. Natural rubber-Its engineering characteristics. Mater. Des. 7, 68-74. http:// www.sciencedirect.com/science/article/pii/ S0261306986800048.

De Snaijer, H., Yuen, J., 2016. Handbook of styrene butadiene rubber. Scitus Acad. LLC.

Dick, J.S., 2011. Basic rubber testing selecting methods for a rubber test program. ASTM International, West Conshohocken, Pa.

Fernández, M.., Gonzáles, N., Mugica, A., Bernicot, C., 2006. Pyrolysis-FTIR and TGA techniques as tools in the characterization of blends of natural rubber and SBR. Thermochem. Acta 1, 65-70.

Ferry, J., Gee, G., Treloar, L.R.., 1945. The interaction between rubber and liquids. VII. The heats and entropies of dilution of natural rubber by various liquids. Rubber Chem. Technol. 19, 1-13.

Flory, P.J., 1942. Constitution of three dimensional polymers and the theory of gelation. J. Phys. Chem. 46, 132-140.

Flory, P.J., Rehner, J., 1943a. Statistical Mechanics of CrossLinked Polymer Networks I. Rubberlike Elasticity. J. Chem. Phys. 11, 512. doi:10.1063/1.1723791

Flory, P.J., Rehner, J., 1943b. Statistical Mechanics of CrossLinked Polymer Networks II. Swelling. J. Chem. Phys. 11, 521. http://link.aip.org/link/JCPSA6/v11/i11/ p521/s1\&Agg=doi. doi:10.1063/1.1723792
Franck, A., Hafner, K., Kern, W.F., 1962. The activation energy of vulcanization. Rubber Chem. Technol. 35, 76-91.

Gee, G., 1943. The interaction between rubber and liquids III. The swellingof vulcanized rubber in various liquids. Rubber Chem. Technol. 16, 263-267.

Gee, G., 1942. The interaction between rubber and liquids II. Thermodynamical basis of the swelling and solution of rubber. Rubber Chem. Technol. 15, 545-552.

Gradwell, M.H.S., Merwe, M.J., 1999a. Reaction of 2-t-butilbenzothiazole sulfenamida with sulfur and zinc oxide in the absence of rubber. Rubber Chem. Technol. $72,55-64$.

Gradwell, M.H.S., Merwe, M.J., 1999b. 2-t-butilbenzotiazole sulfenamide accelerated sulfur vulcanization of polyisoprene. Rubber Chem. Technol. 72, 65-73.

Hauser, E.A., Sze, M.C., 1942. Chemical reactions during vulcanization III. J. Phys. Chem. 46, 118-131.

Heideman, G., 2004. Reduced zinc oxide levels in sulphur vulcanisation of rubber compounds: mechanistic aspects of the role of activators and multifunctional additives. s.n.], S.l.

Heideman, G., Datta, R.N., Noordermeer, J.W.M., Baarle, B.V., 2004. Activators in accelerated sulfur vulcanization. Rubber Chem. Technol. 77, 512-541.

Heinrich, G., Straube, E., Helmis, G., 1988. Rubber elasticity of polymer networks: Theories. Adv. Polym. Sci. 85, 33-87.

Jarny, Y., 2000. SIMULTANEOUS ESTIMATION OF KINETIC PARAMETERS USING GENETIC ALGORITHMS, in: Inverse Problems in Engineering: Theory and Practice: Presented at the 3rd International Conference on Inverse Problems in Engineering, Theory and Practice, June 13-18, 1999, Port Ludlow, Washington. p. 263. http://www.me.ua.edu/3icipe/papers/ paper61.pdf.

Kamal, M.R., Sourour, S., 1973. Kinetics and thermal characterization of thermoset cure. Polym. Eng. Sci. 13, 59-64.

Krishnan, S., 2015. Natural rubber latex filler masterbatch: preparation, processing and evaluation. Sch. Press.

Kuptsov, A.H., Zhizhin, G.N., 1998. Handbook of fourier transform Raman and Infrared spectra of polymers, Elsevier. ed.

Lloyd, D.G., 1991. Additives in rubber processing. Mater. Des. 12, 139-146. http://www.sciencedirect.com/ science/article/pii/026130699190122K. 
Loo, C.T., 1974. High temperature vulcanization of elastomers: 3. Network structure of efficiently vulcanized natural rubber mixes. Polymer 15, 729-737.

Mansilla, M.A., 2012. Influencia de la microestructura en las propiedades mecánicas y térmicas de mezclas de caucho natural y caucho estireno butadieno http:// digital.bl.fcen.uba.ar/Download/Tesis/Tesis_5117_ Mansilla.pdf.

Mansilla, M.A., Quasso, F., Marzocca, A.J., 2007. CARACTERIZACIÓN DE MEZCLAS VULCANIZADAS DE CAUCHO ESTIRENO BUTADIENO Y CAUCHO NATURAL http://www.materiales-org.ar/sitio/biblioteca/CONAMET-SAM2007/sam\%20 conamet $\% 20$ 07 /pdf/T\%C3\%B3pico\%2010\%20-\%20Materiales\%20Polim\%C3\%A9ricos/10-14\%20MansillaM\%20(0).pdf.

Mark, J.E., Erman, B., Roland, M., 2013. The science and technology of rubber (4th ed.). Acad. Press.

Martinez Valdés, M., 2012. Financiamiento, insumo para las empresas productoras de hule natural: procedimientos, riesgos y requisitos para una dispersión de recursos financieros eficiente. Editor. Académica Esp.

Marzocca, A.J., 2007. Evaluation of the polymer-solvent interaction parameter $\chi$ for the system cured styrene butadiene rubber and toluene. Eur. Polym. J. 43, 2682-2689. http://linkinghub.elsevier.com/ retrieve/pii/S0014305707001358. doi:10.1016/j. eurpolymj.2007.02.034

Marzocca, A.J., 2003. Relación entre la cinetica de formación de entrecruzamientos y las propiedades mecánicas de elastomeros vulcanizados. Presented at the Jornadas SAM/CONAMET/Simposio materia, pp. 886-889.

Marzocca, A.J., Cerveny, S., Raimondo, R.B., 1997. Analysis of the variation of molecular parameters of NR during vulcanization in the frame of the conformational tube model. J. Appl. Polym. Sci. 66, 10851092. http://dipc.ehu.es/cerveny/index_htm_files/ journal\% 20 of $\% 20$ polym $\% 20$ sci $\% 2066 \% 20$ (1997)\%201085.pdf.

Marzocca, A.J., Rodriguez Garraza, A.L., Sorichetti, P., Mosca, H.O., 2010. Cure kinetics and swelling behaviour in polybutadiene rubber. Polym. Test. 29, 477-482. http://linkinghub.elsevier.com/retrieve/ pii/S0142941810000280. doi:10.1016/j.polymertesting.2010.02.008
Marzocca, A.J., Steren, C.A., Raimondo, R.B., Cerveny, S., 2004. Influence of the cure level on the monomeric friction coefficient of natural rubber vulcanizates. Polym. Int. 53, 646-655. http://doi.wiley. com/10.1002/pi.1378. doi:10.1002/pi.1378

Mayer, R., 1977. Organic Chemistry of sulfur, S. OAE. ed. Plenum Press, New York.

Morrison, N.J., 1984a. The reactions of crosslink precursors in natural rubber. Rubber Chem. Technol. 57, 86-96.

Morrison, N.J., 1984b. The formation of crosslink precursors in the sulfur vulcanization of natural rubber. Rubber Chem. Technol. 57, 97-103.

Morrison, N.J., Porter, M., 1984a. Temperature effects on the stability of intermediates and crosslinks in sulfur vulcanization. Rubber Chem. Technol. 57, 63-85.

Morrison, N.J., Porter, M., 1984b. Crosslinking of rubbers. Synth. Charact. React. Appl. Polym. G Allen Ed Pergamon Press 57, 115.

Mukhopadhyay, R., Bhowmick, A.K., De, S.K., 1978. Effect of vulcanzation temperature and synergism of accelerators on the network and technical properties of efficiently vulcanized natural rubber mixes. Polymer 19, 1176-1180.

Mukhopadhyay, R., De, S.K., 1977. Effect of vulcanization temperature and vulcanization systems on the structure and properties of natural rubber vulcanizates. Polymer 18, 1243-1249.

Nallasamy, P., Mohan, S., 2004. Vibrational spectra of cis-1,4-Polyisoprene. Arab. J. Sci. Eng. 1A, 17-26.

Ohio, A., 1994. The Science ot Rubber Compounding. Sci. End Technol. Rubber 419. http://books.google.com/ books?hl=en\&lr=\&id=3DRBl2L_lgUC\&oi=fnd \&pg=P A419\&dq=\%22rubber+usage + has + increased + subs tantially+in+modern+radial $\% 22+\% 22$ tire + for $+a+r$ adial+construction+compared+with+approximately $+9+\mathrm{kg}+$ found $\% 22+\% 22$ consumption+is+virtually+i $\mathrm{n}+$ line+with+the+gross+national+product $\% 22+\&$ ot $\mathrm{s}=\mathrm{kr} \_$H4i8Hx2\&sig=lxuYlhqJGtxiwcTo8SU_bs1XRpE.

Ohm, R.F., 1990. The Vanderbilt Rubber Handbook, R.T Vanderbilt Company, INC. ed. Norwalk.

Peres, A.C., Lopes, L.M., Visconte, L.L., Nunes, R.C., 2006. Uso de DSC na determina\ccão de parâmetros de vulcaniza \ccão de látex de borracha natural. Polim. Cienc. E Tecnol. 16, 61. http://www.scielo.br/pdf/ po/v16n1/v16n1a13.pdf. 
Pinchuk, L., Jurkowski, B., Kravtsov, A., Goldade, V., 2001. On some variations in rubber charge state during procesing. Eur. Polym. J. 37, 2239-2243.

Priyadarshan, P.M., 2017. Biology of hevea rubber. Springer. Rabearison, N., Jochum, C., Grandidier, J.C., 2010. A cure kinetics, diffusion controlled and temperature dependent, identification of the Araldite LY556 epoxy. J. Mater. Sci. 46, 787-796. http://link.springer. com/10.1007/s10853-010-4815-7. doi:10.1007/ s10853-010-4815-7

Roland, C.M., 2004. Naval applications of elastomers. Rubber Chem. Technol. 77, 542-551.

Saville, B., Watson, A.A., 1963. Structural characterization of sulfur vulcanizated rubber networks. Rubber Chem. Technol. 36, 547.

Shelton, J.R., McDonel, E.T., 1960. Investigation of radical and polar mechanisms in vulcanization reactions. Rubber Chem. Technol. 33, 342-356.

Urrego, W., Giraldo, D., Álvarez-Láinez, M., 2012. Análisis cuantitativo por FTIR y evaluación comparativa de la descomposición térmica de tres variedades de caucho natural Colombiano. Presented at the SLAP 2012 XIII Simposio latinoamericano de polímeros, Colombia.

Vergnaud, J.M., Rosca, I.-D., 2009. Rubber curing and properties. CRC Press/Taylor \& Francis, Boca Raton. http://www.crcnetbase.com/isbn/978-1-42008522-8.

Villars, D.S., 1957. Studies on carbon black. III. Theory of bound rubber. Rubber Chem. Technol. 30, 157-169.

WHITE, J.L., 1994. Rheological Behavior and Processing ot Unvulcanized Rubber. Sci. End Technol. Rubber 257. http://books.google.com/ books?hl=en\&lr=\&id=3DRBl2L_lgUC\&oi=fnd \&pg=P A257\&dq=\%22and+others, +many+long+forgotten .+It+was+not,+however,+until\%22+\%22\%5BM40, +M41,+M46\%5D+of+the+U.S.+Rubber+Company+ (the+last+two+in $\% 22+\% 22$ compounded+with+la rge+quantities+of+small+particles+exhibited $+\mathrm{a} \% 2$ 2+\&ots=kr_H4i8Hx4\&sig=wbyWW80317XI7pHD0s ElCv3SpYs.

Wren, W.G., 1960. The chemistry of natural rubber production. Rubber Chem. Technol. 12, 378-412.

Zapata, N.C.R., Osswald, T.A., Ortiz, J.P.H., 2012. CINÉTICA DE CURADO DE UN CAUCHO EPDM Y UNA RESINA EPOXÍDICA ALIFÁTICA. MODELAMIENTO Y ANÁLISIS SIN Y CON DIFUSIÓN. Rev. Iberoam. Polímeros 14, 6.

\section{PARA CITAR ESTE ARTÍCULO / TO REFERENCE THIS ARTICLE / PARA CITAR ESTE ARTIGO /}

Urrego-Yepes, W:; Velásquez-Restrepo, S.M.; Giraldo-Vásquez, D.H.; Posada Correa, J.C. (2017). Revisión - Efecto del sistema de vulcanización en la red entrecruzada y en la reacción química de vulcanización del caucho natural. Revista EIA, 14(28), julio-diciembre, pp. 99-115. [Online]. Disponible en: https://doi.org/10.24050/reia.v14i28.1144 